\title{
O ADUBO DE CAMA DE AVIÁRIO NA PRODUÇÃO E NA QUALIDADE DE MUDAS DE EUCALYPTUS DUNNII MAIDEN
}

\author{
The poultry manure fertilizer in the production and in the quality of Eucalyptus dunnii Maiden seedlings
}

\author{
Alexandre Menegatti ${ }^{1}$; Guilherme Oliveira Santos Ferraz de Arruda ${ }^{2}$; Cristiano Nunes Nesi ${ }^{3}$ \\ ${ }^{1}$ Engenheiro Florestal; alexandremenegatti@gmail.com \\ ${ }^{2}$ Docente; Curso de Engenharia Florestal; Universidade do Oeste de Santa Catarina; campus Xanxerê SC; \\ guilherme.arruda@unoesc.edu.br \\ ${ }^{3}$ Docente; Cursos de Engenharia Florestal e Agronomia; Universidade do Oeste de Santa Catarina; campus Xanxerê SC; \\ cristiano.nesi@unoesc.edu.br
}

Artigo enviado em 18/02/2017, aceito em 26/03/2017 e publicado em 11/04/2017.

\begin{abstract}
Resumo - A cama de aviário é um resíduo orgânico abundante provindo da criação de aves, do qual se obtêm um adubo que pode ser utilizado na produção de mudas de espécies florestais. O objetivo deste estudo foi avaliar o efeito do adubo de cama de aviário na germinação, desenvolvimento e qualidade de mudas de Eucalyptus dunnii Maiden em casa de vegetação. $\mathrm{O}$ delineamento experimental foi inteiramente casualizado, com cinco tratamentos e quatro repetições. Os tratamentos foram $0 \%, 20 \%, 40 \%, 60 \%$ e $80 \%$ de adubo de cama de aviário, adicionados ao substrato comercial à base de turfa e casca de arroz carbonizada. Foram avaliados periodicamente (30,60 e 90 dias) o índice de velocidade de germinação, índice de velocidade de emergência, diâmetro de coleto, altura, relação entre altura e diâmetro do coleto e a sobrevivência das mudas. Aos 90 dias avaliaram-se o índice de qualidade de Dickson e os pesos de matéria seca das raízes, da parte aérea e total. O adubo de cama de aviário é um produto viável para produção de mudas de Eucalyptus dunnii em quantidades moderadas. O uso de $20 \%$ de adubo de cama de aviário no volume total do substrato favorece o desenvolvimento e a qualidade das mudas. Quantidades superiores a $20 \%$ tendem a ser prejudiciais à germinação e à sobrevivência das mesmas.
\end{abstract}

Palavras-Chave - resíduo orgânico, substrato, espécies florestais, casa de vegetação.

\begin{abstract}
The poultry manure is an abundant organic residue from the poultry farming, from which a fertilizer can be obtained and used in the production of forest species seedlings. The objective of this study was to evaluate the effect of the poultry manure fertilizer on germination, development and quality of Eucalyptus dunnii Maiden seedlings in a greenhouse. The experimental design was completely randomized, with five treatments and four repetitions. The treatments were $0 \%, 20 \%$, $40 \%, 60 \%$ e $80 \%$ of poultry manure fertilizer added on commercial substrate made of peat and charred rice husk. Periodically (30, 60 and 90 days) the germination speed index, the emergence speed index, the stem diameter, height, the relation between height and stem diameter and the survival of seedlings were evaluated. At 90 days were evaluated the Dickson quality index, dry weight of root, dry weight of shoot and dry weight total. The poultry manure fertilizer is a viable product for use in the production of Eucalyptus dunnii seedlings in moderate amounts. The use of $20 \%$ of poultry manure fertilizer in the total volume of the substrate favors the development and quality of the seedlings. Quantities greater than $20 \%$ tend to be harmful to germination and their survival.
\end{abstract}

Keywords - organic residue, substrate, forest species, greenhouse. 


\section{INTRODUÇÃO}

Materiais orgânicos diversos podem ser utilizados com sucesso como fertilizante ou em misturas na composição de substratos para culturas florestais e agrícolas. Os resíduos vegetais e dejetos animais, devidamente tratados, constituem importantes fontes nutricionais disponíveis para adubação orgânica. Estes apresentam sustentabilidade por promover o retorno dos nutrientes ao solo (CORREAA; MIELE, 2011) assim como ganham importância por aproveitar resíduos, reduzir o impacto ambiental, possibilitar o aumento no rendimento das culturas comerciais e reduzir o custo de produção ao substituir a adubação mineral (FÁVERO, 2012).

A produção de mudas florestais, como as do gênero Eucalyptus, tem se beneficiado de insumos produzidos de origem vegetal e animal, geralmente na composição de substratos para viveiros florestais. Este gênero florestal, amplamente difundido no segmento florestal, tem ocorrência natural na Austrália e grande capacidade de adaptação, sendo largamente utilizado em plantios florestais. A introdução do eucalipto no Brasil deu-se para suprir as demandas por lenha, postes e dormentes para ferrovias. Atualmente, seu plantio destinase à produção de produtos sólidos de madeira (chapas, painéis, roliça), celulose e papel, energia de biomassa (lenha e carvão), entre outros (LANG; BOTREL, 2008; LONGUE JÚNIOR; COLODETTE, 2013).

No entanto, poucas espécies deste gênero apresentam aptidão para regiões com ocorrência de geadas, como nos estados do sul do Brasil. Neste contexto, o Eucalyptus dunnii Maiden destaca-se como uma das alternativas ao plantio. O E. dunnii é uma espécie que apresenta grandes vantagens quanto aos aspectos silviculturais (IWAKIRI et al., 2007). Com destaque pelo seu rápido crescimento, uniformidade dos talhões, forma das árvores e adapta-se satisfatoriamente às regiões de inverno rigoroso com geadas (FLORSHEIM et al., 2009). Entretanto, assim como outras espécies do mesmo gênero indicadas para regiões subtropicais, apresenta baixo índice de enraizamento, o que dificulta a propagação vegetativa e direciona para a produção de mudas via sementes. Desta forma, a importância de se conhecer o que afeta a formação de raízes e suas implicações está relacionada ao sucesso ou ao fracasso da produção de mudas (BRONDANI et al., 2010).

A pressão para o uso de substratos renováveis é uma tendência que encontra-se relacionada à busca pela qualidade das mudas florestais, cuja determinação baseiase em aspectos fisiológicos e morfológicos. Os fisiológicos são em geral mais difíceis de se mensurar, sendo o índice de velocidade de germinação um dos parâmetros mais utilizados. Os morfológicos são mais utilizados na determinação da qualidade das mudas florestais, pela facilidade de mensuração da altura, diâmetro do coleto, relação altura/diâmetro do coleto, peso seco de biomassa aérea e de raízes e índice de qualidade de Dickson (GOMES; PAIVA, 2011).
O uso de substratos apropriados é essencial para assegurar a boa adaptação e crescimento das mudas após o plantio (DEL QUIQUI et al., 2004). O substrato é um dos fatores que influenciam na qualidade das mudas, provendo condições para o desenvolvimento do sistema radicular.

Devido à maior pressão para o uso de substratos não agressivos ao ambiente durante sua extração, buscamse materiais que sejam oriundos de resíduos renováveis (WENDLING; GUASTALA; DEDECEK, 2007).

A adubação é outro fator fundamental para produzir mudas em boa quantidade e qualidade. Em viveiros florestais costuma-se utilizar dois métodos de fertilização: via cobertura (por irrigação) e na base (incorporado ao substrato) (SILVA et al., 2013). De acordo com De Marco et al. (2013) o fornecimento de doses adequadas de nutrientes, de origem orgânica ou mineral, reflete diretamente no desenvolvimento e crescimento de espécies florestais.

Diversos materiais orgânicos podem ser utilizados como fertilizante em mistura com substratos. Os resíduos de culturas agrícolas e o esterco de animais constituem importantes fontes disponíveis desta adubação orgânica.

O sistema de integração entre pequenas propriedades rurais e as agroindústrias, possibilitou à avicultura ganhar espaço e com ela há produção em grande escala de cama de aviário (VALMORBIDA; FUNEZ, 2011), da qual pode-se obter o respectivo adubo para uso agrícola e florestal. A cama de aviário apresenta também a vantagem de liberar lentamente os nutrientes, podendo assim coincidir com a maior demanda nutricional da cultura (CORRÊA; MIELE, 2011). O adubo de cama de aviário, normalmente apresenta elevados teores de nutrientes, como N, P, K e Ca, superiores ao esterco de gado, outra fonte de adubo orgânico muito utilizada (OLIVEIRA et al., 2008).

O objetivo deste estudo foi avaliar o efeito de proporções de cama de aviário em mistura com substrato comercial na germinação, desenvolvimento e qualidade de mudas de E. dunnii.

\section{MATERIAL E MÉTODOS}

O experimento foi conduzido em casa de vegetação não climatizada da Universidade do Oeste de Santa Catarina - UNOESC Campus Xanxerê, situada a $800 \mathrm{~m}$ de altitude, de janeiro a abril de 2015. O clima na região é mesotérmico úmido com verão quente, do tipo Cfa (Koeppen), precipitação média $2100 \mathrm{~mm} / \mathrm{ano}$, temperatura média entre $16^{\circ} \mathrm{C}$ e $17^{\circ} \mathrm{C}$. As mudas foram produzidas a partir de sementes peletizadas (Lote: J19072013) adquiridas em estabelecimento comercial especializado em Rio do Sul, SC e provenientes de área de produção de sementes (APS). A semeadura foi realizada em tubetes cônicos $\left(100 \mathrm{~cm}^{3}\right)$ dispostos em mesas metálicas para tubetes de 1,0 m x 1,0 m. As irrigações, via 
aspersão, foram programadas para três minutos durante quatro vezes ao dia.

Utilizou-se substrato comercial para mudas florestais, composto por turfa, casca de arroz carbonizada, calcário calcítico e aditivado com N (0,04\%), $\mathrm{P}_{2} \mathrm{O}_{5}(0,04 \%)$ e $\mathrm{K}_{2} \mathrm{O}(0,05 \%)$. O adubo de cama de aviário, já curtido e peneirado, foi obtido em empresa de comércio atacadista de fertilizantes orgânicos em Chapecó/SC.

Os tratamentos aplicados foram constituídos de adubo obtido de cama de aviário, adicionado a um substrato comercial para mudas florestais, nas seguintes proporções: $100 \%$ substrato (T1); $80 \%$ de substrato + $20 \%$ de adubo de aviário (T2); $60 \%$ de substrato $+40 \%$ de adubo de aviário (T3); 40\% de substrato $+60 \%$ de adubo de aviário (T4) e $20 \%$ de substrato $+80 \%$ de adubo de aviário (T5).

O delineamento experimental foi inteiramente casualizado, com cinco tratamentos, 20 mudas por tratamento e quatro repetições.

Os tratamentos foram avaliados quanto à germinação e emergência. Para obtenção do Índice de Velocidade de Germinação (IVG) e do Índice de Velocidade de Emergência (IVE) foram feitas observações diárias com contagem do número de sementes germinadas e de plântulas emergidas. O IVG foi obtido por:

$$
I V G=\sum \frac{G_{n}}{N_{n}}
$$

Em que $G_{n}$ é o número de sementes germinadas e $\mathrm{N}_{\mathrm{n}} \mathrm{O}$ número de dias decorridos da semeadura. O IVE foi obtido por:

$$
I V E=\sum \frac{E_{n}}{N_{n}}
$$

Em que $E_{n}$ é o número de mudas emergidas e $\mathrm{N}_{\mathrm{n}} \mathrm{o}$ número de dias decorridos da semeadura. As sementes foram consideradas germinadas após a exposição do hipocótilo e emergidas após a formação do primeiro par de folhas não cotiledonares.

As mudas também foram avaliadas aos $30,60 \mathrm{e}$ 90 dias quanto aos parâmetros altura $(\mathrm{H})$ e diâmetro de coleto (DC). A altura das mudas foi mensurada com o uso de régua a partir do nível do substrato até a ponta da última folha, enquanto o diâmetro de coleto foi mensurado com paquímetro digital com leitura em milímetros. Aos 60 dias ampliou-se o espaçamento entre as mudas nas mesas metálicas para $4,5 \mathrm{~cm}$ x $4,5 \mathrm{~cm}$ para melhor desenvolvimento. As mudas foram avaliadas ainda quanto à porcentagem de sobrevivência.

Aos 90 dias fez-se a pesagem da matéria seca total (PMST), da matéria seca de raízes (PMSR) e de matéria seca da parte aérea (PMSPA) das mudas. Para isso, as mudas foram retiradas dos tubetes, lavadas em água corrente e separadas as raízes e partes aéreas. Posteriormente, ambas as partes foram secas em estufa a $75^{\circ} \mathrm{C}$ por 72 horas e imediatamente pesadas em balança analítica (GOMES et al., 2002). A avaliação incluiu a relação entre a altura e o diâmetro de coleto (H/DC), bem como o Índice de Qualidade de Dickson (IQD) (GOMES; PAIVA, 2011), o qual é determinado em função da altura $(\mathrm{H})$, diâmetro do peso de matéria seca da parte aérea (PMSPA), peso de matéria seca das raízes (PMSR) e peso de matéria seca total (PMST), utilizando-se a equação:

$$
I Q D=\frac{P M S T(g)}{\frac{H(\mathrm{~cm})}{D C(\mathrm{~mm})}+\frac{P M S P A(g)}{P M S R(g)}}
$$

Os dados foram submetidos à análise estatística com programa R (R DEVELOPMENT CORE TEAM, 2011). Inicialmente, realizou-se o teste de Bartlett para homogeneidade de variâncias entre os tratamentos, assim como o teste de Shapiro Wilk, em que avaliou-se a distribuição normal dos resíduos. Nos casos em que foram verificadas variâncias heterogêneas e/ou distribuição não normal de resíduos, realizou-se transformação dos dados de acordo com o parâmetro de transformação lambda $(\boldsymbol{\lambda})$ de Box-Cox (BOX; COX, 1964). Atendidas as pressuposições de homogeneidade de variâncias e de distribuição normal dos resíduos prosseguiu-se com a Análise de Variância onde se verificou efeito de tratamentos a $5 \%$ de probabilidade. Por fim, realizou-se o teste Scott-Knott a 5\% de probabilidade para verificar as diferenças entre as médias dos tratamentos.

\section{RESULTADOS E DISCUSSÃO}

Devido ao efeito negativo de altas doses do adubo de cama de aviário na germinação, optou-se pela eliminação de T5. Este tratamento apresentou valores muito distintos dos demais. Conforme mencionam Banzatto e Kronka (2006) é possível eliminar tratamentos com valores discrepantes. O percentual de germinação neste tratamento não ultrapassou 15\%, dificultando a estimativa de médias dos parâmetros.

O início das germinações deu-se no sexto dia após a semeadura, situação semelhante ao encontrado por Cetnarski Filho e Carvalho (2009), os quais avaliaram o tempo médio de germinação para o E. dunnii. Garcia et al. (2011) verificaram o início das germinações desta mesma espécie no quinto dia após a semeadura. De forma semelhante ao presente estudo, estes autores observaram que a presença do adubo de cama de aviário propiciou maior atraso na germinação, o que influencia diretamente o IVG.

A Tabela 1 apresenta as médias dos tratamentos para o índice de velocidade de germinação (IVG), índice de velocidade de emergência (IVE) e sobrevivência. Podese observar que T1 e T2 apresentaram diferença significativa de T3 e T4 para estes parâmetros. Nota-se também que o gradual acréscimo no percentual de adubo de cama de aviário de cada tratamento propiciou uma 
redução gradativa nas médias nos parâmetros IVG, IVE e sobrevivência.

Tabela 1: Médias do Índice de Velocidade de Germinação (IVG), Índice de Velocidade de Emergência (IVE) e sobrevivência para mudas de $E$. dunnii em doses crescentes de cama de aviário.

Table 1: Average of Germination Speed Index (GSI), Emergency Speed Index (ESI) and survival for E. dunnii seedlings with increasing doses of poultry manure.

\begin{tabular}{cccc}
\hline Tratamento & IVG & IVE $^{1}$ & $\begin{array}{c}\text { Sobrevivência } \\
(\%)\end{array}$ \\
\hline T1 & $33,86 \mathrm{a}$ & $25,80 \mathrm{a}$ & $97,50 \mathrm{a}$ \\
T2 & $29,97 \mathrm{a}$ & $22,55 \mathrm{a}$ & $96,25 \mathrm{a}$ \\
T3 & $22,57 \mathrm{~b}$ & $12,88 \mathrm{~b}$ & $86,25 \mathrm{~b}$ \\
T4 & $18,78 \mathrm{~b}$ & $8,88 \mathrm{~b}$ & $86,25 \mathrm{~b}$ \\
\hline
\end{tabular}

* Médias seguidas da mesma letra não diferem entre si pelo teste ScottKnott a $5 \%$ de probabilidade de erro. Médias apresentadas em escala original.

${ }^{1}$ Transformação: Log x. (Transformações de acordo com Lambda de Box-Cox).

Estes resultados demonstram que a utilização de grandes doses de adubo de cama de aviário junto ao substrato é prejudicial à germinação das sementes de E. dunnii. Cetnarski Filho e Carvalho (2009) avaliaram índices de velocidade de germinação (IVG) de E. dunnii em substratos e obtiveram valores médios de 27,54 para substrato de areia, 22,40 para substrato de papel e 20,30 para substrato de vermiculita.

Para o IVE observou-se um efeito prejudicial das maiores doses de adubo de cama de aviário. Também Brugnara (2014) verificou que a adição de cama de aviário ao substrato comercial reduziu o IVE de mudas de Passiflora edulis Sims f. flavicarpa Deg. Já Sorgato et al. (2014) avaliaram o IVE de Tropaeolum majus e não observaram diferença significativa com a utilização de cama de aviário incorporada ao substrato.

A sobrevivência das mudas também apresentouse comprometida pela utilização das maiores doses de adubo de aviário. Em semelhança ao presente estudo De Marco et al. (2013) observaram que o aumento da quantidade de cama de aviário no substrato para mudas Toona ciliata M. Roem foi prejudicial à sobrevivência das mesmas.

A Tabela 2 apresenta as médias dos tratamentos para altura das mudas mensuradas aos 30,60 e 90. Aos 30 dias observou-se um melhor desempenho do tratamento T2, o qual apresentou média superior. Já T1, T3 e T4 não diferiram entre si. Essa mesma tendência foi verificada aos 60 dias, com exceção de T1 que demonstrou menor eficiência para este parâmetro. Aos 90 dias, as médias de T2, T3 e T4 passaram a não diferir significativamente entre si, mas diferiram do T1 que apresentou a menor média.
Tabela 2: Altura (H) das mudas de E. dunnii aos 30, 60 e 90 dias em doses crescentes de cama de aviário.

Table 2: Height $(\mathrm{H})$ of $E$. dunnii seedlings at 30, 60 and 90 days with increasing doses of poultry manure.

\begin{tabular}{cccc}
\hline Tratamento & $\mathrm{H}^{1} 0^{1}(\mathrm{~cm})$ & $\mathrm{H} 60(\mathrm{~cm})$ & $\mathrm{H} 90^{2}(\mathrm{~cm})$ \\
\hline T1 & $2,88 \mathrm{~b}$ & $6,56 \mathrm{c}$ & $9,59 \mathrm{~b}$ \\
$\mathrm{~T} 2$ & $4,65 \mathrm{a}$ & $18,84 \mathrm{a}$ & $25,57 \mathrm{a}$ \\
$\mathrm{T} 3$ & $2,28 \mathrm{~b}$ & $14,03 \mathrm{~b}$ & $21,41 \mathrm{a}$ \\
$\mathrm{T} 4$ & $2,11 \mathrm{~b}$ & $15,09 \mathrm{~b}$ & $22,32 \mathrm{a}$ \\
\hline
\end{tabular}

* Médias seguidas da mesma letra não diferem entre si pelo teste ScottKnott a $5 \%$ de probabilidade de erro. Médias apresentadas em escala original.

${ }^{1}$ Transfomação: $\log \mathrm{x} .{ }^{2}$ Transformação $\mathrm{x}^{-1}$. (Transformações de acordo com Lambda de Box-Cox).

Em estudo realizado por Kratz e Wendling (2013), avaliando diferentes substratos também em mudas de E. dunnii, os maiores valores observados para altura das mudas aos 90 dias foram de 16,02 e 13,15 cm. Valores próximos foram encontrados para o presente trabalho aos 60 dias. Aos 90 dias observou-se uma média de 25,57 cm para T2, o que aponta para a eficiência da cama de aviário no incremento deste parâmetro. Utilizando substrato comercial à base de turfa e casca de arroz carbonizada, Ehlers e Arruda (2014) obtiveram valor médio de altura aos 180 dias de $19,60 \mathrm{~cm}$ para Eucalyptus grandis ao avaliarem o efeito do pó de basalto no desenvolvimento das mudas.

A Tabela 3 apresenta médias dos tratamentos para o diâmetro de coleto aos 30,60 e 90 dias. Pode-se notar que aos 30 dias o T2 apresentou média superior com diferença significativa em relação aos demais tratamentos. Tanto aos 60, como 90 dias, apesar de apresentar média numericamente superior, $\mathrm{O}$ T2 não diferiu significativamente de T3 e T4. O T1, por sua vez, embora com valores inferiores aos 30, 60 e 90 dias para este parâmetro, diferiu significativamente dos demais tratamentos aos 60 e 90 dias. 
Tabela 3: Diâmetro de Coleto (DC) das mudas de E. dunnii aos 30, 60 e 90 dias em doses crescentes de cama de aviário. Table 3: Stem diameter (ST) of $E$. dunnii seedlings at 30, 60 and 90 days with increasing doses of poultry manure.

\begin{tabular}{cccc}
\hline Tratamento & $\begin{array}{c}\text { DC30 } \\
(\mathrm{mm})\end{array}$ & $\begin{array}{c}\text { DC60 } \\
(\mathrm{mm})\end{array}$ & $\begin{array}{c}\text { DC90 } \\
(\mathrm{mm})\end{array}$ \\
\hline T1 & $0,53 \mathrm{~b}$ & $1,04 \mathrm{~b}$ & $1,19 \mathrm{~b}$ \\
$\mathrm{~T} 2$ & $0,68 \mathrm{a}$ & $3,94 \mathrm{a}$ & $2,24 \mathrm{a}$ \\
$\mathrm{T} 3$ & $0,48 \mathrm{~b}$ & $1,39 \mathrm{a}$ & $2,07 \mathrm{a}$ \\
$\mathrm{T} 4$ & $0,46 \mathrm{~b}$ & $1,43 \mathrm{a}$ & $2,09 \mathrm{a}$ \\
\hline
\end{tabular}

* Médias seguidas da mesma letra não diferem entre si pelo teste ScottKnott a $5 \%$ de probabilidade de erro. Médias apresentadas em escala original.

${ }^{1}$ Transformação: $x^{-2}$. (Transformações de acordo com Lambda de BoxCox).

Ao avaliarem diferentes substratos para esta mesma espécie, Kratz e Wendling (2013) observaram valores para o diâmetro de coleto aos 90 dias entre $0,99 \mathrm{e}$ $1,79 \mathrm{~mm}$. Trazzi et al. (2013) verificaram o efeito da utilização de estercos de animais como componente de substrato na produção de mudas de Tectona grandis Linn. F. e concluíram que a cama de frango também proporcionou maiores ganhos no diâmetro de coleto das mudas.

Na Tabela 4 estão compiladas as médias para o parâmetro H/DC aos 30, 60 e 90 dias nos diversos tratamentos. Conforme Gomes e Paiva (2011), a relação H/DC é um importante índice de qualidade para mudas florestais e quanto menor for seu valor numérico, maior será a capacidade das mudas sobreviverem e se estabelecerem na área com sucesso após o plantio.

Tabela 4. Relação H/DC das mudas de E. dunnii aos 30, 60 e 90 dias em doses crescentes de cama de aviário.

Table 4. H / SD ratio of E. dunnii seedlings at 30,60 and 90 days with increasing doses of poultry manure.

\begin{tabular}{cccc}
\hline Tratamento & H/DC 30 & H/DC 60 & H/DC 90 \\
\hline T1 & $5,23 \mathrm{a}$ & $7,24 \mathrm{a}$ & $7,97 \mathrm{a}$ \\
T2 & $6,55 \mathrm{~b}$ & $10,93 \mathrm{~b}$ & $11,39 \mathrm{~b}$ \\
T3 & $4,55 \mathrm{a}$ & $9,90 \mathrm{~b}$ & $10,40 \mathrm{~b}$ \\
T4 & $4,31 \mathrm{a}$ & $11,40 \mathrm{~b}$ & $10,77 \mathrm{~b}$ \\
\hline
\end{tabular}

* Médias seguidas da mesma letra não diferem entre si pelo teste Scott-Knott a 5\% de probabilidade de erro. Médias apresentadas em escala original.

${ }^{1}$ Transformação: $\mathrm{x}^{-3}$. (Transformações de acordo com Lambda de Box-Cox).

Aos 30 dias, observou-se que os tratamentos T4, T3 e T1 apresentaram respectivamente os valores médios mais baixos e, portanto, os melhores resultados para a idade. O T2 diferiu significativamente com o maior valor médio. Já aos 60 e 90 dias as melhores médias foram do T1, que diferiram estatisticamente dos demais tratamentos. Para Carneiro (1995) a relação H/DC deve situar-se entre 5,4 e 8,1, entretanto para Kratz e Wendling (2013) esta faixa não é a mais indicada para o eucalipto e encontraram valores para H/DC entre 6,52 e 8,95 ao avaliarem mudas de E. dunnii aos 90 dias produzidas com substratos a base de casca de pinus, casca de arroz carbonizada, vermiculita e fibra de coco.

$\mathrm{Na}$ Tabela 5 são apresentados os valores médios do IQD para os tratamentos. Contraditoriamente aos demais parâmetros, o T4 apresentou a maior média, o que, conforme Gomes e Paiva (2011), indica melhor padrão de qualidade das mudas. Entretanto, este tratamento não diferiu estatisticamente de T2 e T3. Apenas T1 diferiu dos demais tratamentos e seu menor valor indica sua menor qualidade.

Tabela 5. Índice de Qualidade de Dickson (IQD) para os tratamentos em $E$. dunni em doses crescentes de cama de aviário.

Table 5. Dickson Quality Index (DQI) for treatments in $E$. dunnii with increasing doses of poultry manure.

\begin{tabular}{cc}
\hline Tratamento & IQD $^{1}$ \\
\hline T1 & $0,0268 \mathrm{~b}$ \\
T2 & $0,1019 \mathrm{a}$ \\
T3 & $0,1044 \mathrm{a}$ \\
T4 & $0,1150 \mathrm{a}$ \\
\hline
\end{tabular}

* Médias seguidas da mesma letra não diferem entre si pelo teste ScottKnott a $5 \%$ de probabilidade de erro. Médias apresentadas em escala original.

${ }^{1}$ Transformação ${ }^{-0,5}$. (Transformações de acordo com Lambda de BoxCox).

Resultados semelhantes para o E. dunnii foram observados por Pichler (2011) ao avaliar o uso de Biofertilizante Supermagro ${ }^{\circledR}$. Esses autores em avaliação aos 120 dias, com aplicação deste fertilizante a cada 14 dias, em concentrações de $10 \%$ e $15 \%$, encontraram, para ambas as concentrações, valores de 0,10 para o IQD.

A Tabela 6 contém as médias dos tratamentos para o peso de matéria seca das raízes, da parte aérea e peso total das mudas aos 90 dias. Pode-se notar que os três parâmetros apresentaram a mesma tendência. T2, T3 e T4 apresentam médias superiores, porém não diferem estatisticamente entre si. Apenas T1 apresentou média com diferença significativa, entretanto com valor inferior, indicando menor ganho de matéria seca deste tratamento em comparação aos demais. 
Tabela 6. Peso de material seca das raízes (PMSR), peso de matéria seca da parte aérea (PMSPA) e peso de matéria seca total (PMST) para E. dunnii em doses crescentes de cama de aviário.

Table 6. Roots dry matter weight (RDMW), aerial part dry matter weight (APDMW) and total dry matter weight (TDMW) for $E$. dunnii with increasing doses of poultry manure.

\begin{tabular}{cccc}
\hline Tratamento & PMS R (g) & PMS PA $^{1}(\mathrm{~g})$ & PMS T $^{2}(\mathrm{~g})$ \\
\hline T1 & $0,1119 \mathrm{~b}$ & $0,1378 \mathrm{~b}$ & $0,2497 \mathrm{~b}$ \\
T2 & $0,4179 \mathrm{a}$ & $0,9982 \mathrm{a}$ & $1,4161 \mathrm{a}$ \\
T3 & $0,3740 \mathrm{a}$ & $0,9763 \mathrm{a}$ & $1,3503 \mathrm{a}$ \\
T4 & $0,4092 \mathrm{a}$ & $1,1521 \mathrm{a}$ & $1,5613 \mathrm{a}$ \\
\hline
\end{tabular}

* Médias seguidas da mesma letra não diferem entre si pelo teste ScottKnott a 5\% de probabilidade de erro. Médias apresentadas em escala original.

${ }^{1}$ Transformação $\mathrm{x}^{-1} \cdot{ }^{2}$ Transformação $\mathrm{x}^{-0,5}$. (Transformações de acordo com Lambda de Box-Cox).

De forma semelhante ao presente estudo Lang e Botrel (2008) obtiveram média com valor superior para o peso de matéria seca da parte aérea ao utilizar substrato comercial com cama de aviário como substrato em mudas de Eucalyptus grandis. De Marco et al. (2013) verificaram que a cama de aviário incorporada ao substrato para produção de mudas de Toona ciliata possui tendência em promover um aumento no peso de matéria seca das raízes.

\section{CONCLUSÕES}

O adubo de cama de aviário é um componente viável para a produção de mudas de E. dunnii em quantidades moderadas.

A utilização de $20 \%$ de adubo de cama de aviário no substrato comercial à base de turfa e casca de arroz carbonizada favorece o desenvolvimento e a qualidade das mudas de E. dunnii.

Quantidades maiores que 20\% de adubo de cama de aviário no substrato comercial à base de turfa e casca de arroz carbonizada interferem negativamente na germinação e na sobrevivência de E. dunnii.

\section{REFERÊNCIAS}

BANZATTO, D. A.; KRONKA, S. N. Experimentação agricola. Jaboticabal: Funep, 2006. 237 p.

BOX, G. E. P.; COX, D. R. An analysis of transformations. Journal of the Royal Statistical Society, Chichester, p. 211-252, 1964.

BRONDANI, G. E.; GROSSI, F.; WENDLING, I.; DUTRA, L. F.; ARAÚJO, M. A. Aplicação de IBA para o enraizamento de miniestacas de Eucalyptus benthamii Maiden \& Cambage x Eucalyptus dunnii Maiden. Acta
Scientiarum Agronomy, Maringá, v. 32, n. 4, p. 667-674, 2010.

BRUGNARA, E. C. Cama de aviário em substratos para mudas de maracujazeiro-amarelo. Revista Brasileira de Agroecologia. v. 9, n. 3, p. 21-30, 2014.

CARNEIRO, J. G. de A. Produção e controle de qualidade de mudas florestais. Curitiba: UFPR/FUPEF, 1995. 451p.

CETNARSKI FILHO, R.; CARVALHO, R. I. N. de. Massa da amostra, substrato e temperatura para teste de germinação de sementes de Eucalyptus dunnii Maiden. Ciência Florestal, Santa Maria, v. 19, n. 3, p. 257-265, jul./set. 2009.

CORRÊA, J. C.; MIELE, M. Manejo Ambiental na Avicultura. In: PALHARES, J. C. P.; KUNZ, A. (Ed.). A cama de aves e os aspectos agronômicos, ambientais e econômicos. Documentos: 149, Embrapa Suínos e Aves. Concórdia, 221 p. 2011.

DE MARCO, R.; CONTE, B.; PERRANDO, E. R.; FORTES, F. O.; MARTARELLO, V. Resposta de mudas de Toona ciliata M. Roem à fertilização orgânica e química. Enciclopédia Biosfera, Goiânia, v.9, n.16, p. 1939, 2013.

DEL QUIQUI, E. M.; MARTINS, S. S. M.; PINTRO, J. C.; ANDRADE, P. J. P. de; MUNIZ, A. S. Crescimento e composição mineral de mudas de eucalipto cultivadas sob condições de diferentes fontes de fertilizantes. Acta Scientiarum, Maringá, v. 26, n. 3, p. 293-299, 2004.

EHLERS, T.; ARRUDA, G. O. S. F. de. Utilização do Pó de Basalto em Substratos para Mudas de Eucalyptus grandis. Floresta e Ambiente, Seropédica, v. 21, n. 1, p. 37-44, jan./mar. 2014.

FÁVERO, F. Uso da cama de frango associada à adubação mineral no sistema de produção de grãos da região oeste do Paraná. 2012. 79 f. Dissertação (Mestrado em Agronomia) Universidade Estadual do Oeste do Paraná. Marechal Cândido Rondon, 2012.

FLORSHEIM, S. M. B.; COUTO, H. T. Z. do; LIMA, I. L. de; LONGUI, E. L. Variação nas dimensões dos elementos anatômicos da madeira de Eucalyptus dunnii aos sete anos de idade. Revista do Instituto Florestal, São Paulo, v. 21, n. 1, p. 79-91, jun. 2009.

GARCIA, K. R. L.; FERREIRA, P. J. F.; GUERREIRO, J. C.; PETRELI, D. da S.; ZANZARINI, M. B. Utilização de Substratos Alternativos na Produção de Mudas de Eucalyptus dunni. In: X Congresso da Iniciação Científica. Anais... Faculdades Integradas de Ourinhos, Ourinhos, SP, 2011. 
GOMES, J. M.; COUTO, L.; LEITE, H. G.; XAVIER, A.; GARCIA, S. L. R. Parâmetros morfológicos na avaliação da qualidade de mudas de Eucalyptus grandis. Revista Árvore, Viçosa, v.26, n.6, p.655-664, 2002.

GOMES, J. M.; PAIVA, H.N. Viveiros florestais: Propagação Sexuada. Viçosa: Editora UFV, 2011. 116 p.

IWAKIRI, S.; KEINERT JR, S.; PRATA, J. G.; ROSSO, S. Produção de painel compensado estrutural de Eucalyptus grandis e Eucalyptus dunnii. Floresta, Curitiba, v. 37, n. 3, p. 363-367, set./dez. 2007.

KRATZ, D.; WENDLING, I. Produção de mudas de Eucalyptus dunnii em substratos renováveis. Floresta, Curitiba, PR, v. 43, n. 1, p. 125 - 136, jan./mar. 2013.

LANG, D. Z.; BOTREL, C. G. B. Desenvolvimento de mudas de Eucalyptus grandis Hill ex Maiden em diferentes substratos. Cultivando o Saber, Cascavel, v.1, n.1, p.107-117, 2008.

LONGUE JÚNIOR, D.; COLODETTE, J. L.

Importância e versatilidade da madeira de eucalipto para a indústria de base florestal. Pesquisa Florestal Brasileira, Colombo, v. 33, n. 76, p. 429-438, out./dez. 2013.

OLIVEIRA, F. L. de; GUERRA, J. G. M.; ALMEIDA, D. L.; RIBEIRO, R. L. D.; SILVA, E. D.; SILVA, V. V.; ESPINDOLA, J. A. A. Desempenho de taro em função de doses de cama de aviário, sob sistema orgânico de produção. Horticultura Brasileira, Brasília, v. 26, n. 2, p.149153, 2008.

PICHLER, M. E. B. Avaliação do biofertilizante supermagro na produção de mudas de Eucalyptus dunnii Maiden. 2011, 66 f. Dissertação (Mestrado em Ciências Florestais) -

Universidade Estadual do Centro Oeste, Irati, PR, 2011.

R DEVELOPMENT CORE TEAM. R: A Language and Environment for Statistical Computing, R Foundation for Statistical Computing, Vienna, Austria, 2011.

SILVA, R. B. G. da.; SIMÕES, D.; ANDRADE, F. R.; SILVA, M. R. da. Qualidade de mudas seminais de eucalipto em função dos substratos e fertilização de liberação controlada. Interciencia, Caracas, v. 38, p. 215 220, 2013.

SORGATO, J. C.; ROSA, D. B. C. J.; MORENO, L. B.; SOARES, J. S.; VIEIRA, M. C. Emergência de Capuchinha em diferentes substratos. Enciclopédia Biosfera, Goiânia, v. 10, n. 19, p. 953, 2014.

TRAZZI, P. A.; CALDEIRA, M. V. W.; PASSOS, R. R.; GONÇALVES, E. O. Substratos de origem orgânica para produção de mudas de Teca (Tectona grandis Linn. F.).
Ciência Florestal, Santa Maria, v. 23, n. 3, p. 401-409, jul./set. 2013.

VALMORBIDA, F. D. L.; FUNEZ, L. M.

Sustentabilidade econômica e ambiental da atividade avícola em propriedades do município de Itá - SC. Ágora, Mafra, v. 18, n. 1, p 35 - 43, 2011.

WENDLING, I.; GUASTALA, D; DEDECEK, R. Características físicas e químicas de substratos para produção de mudas de Ilex paraguariensis St.-Hil. Árvore, Viçosa, v. 31, p. 209 - 220, 2007. 